\title{
DISTRIBUTION AND MANAGEMENT OF THE INVASIVE EXOTIC SPECIES AMBROSIA TRIFIDA AND SICYOS ANGULATUS IN THE SEOUL METROPOLITAN AREA
}

\section{Kee Dae Kim ${ }^{1}$}

${ }^{1}$ Department of Environmental Education, Korea National University of Education, Cheongju 361-892, Republic of Korea, e-mail: kdkim@knue.ac.kr

Received: 2017.07.08 Accepted: 2017.08.01 Published: 2017.09.01

\begin{abstract}
We investigated the status of invasive exotic plants disturbing the ecosystem of the Seoul metropolitan area and examined the management of such plants. We selected our study sites based on those used in previous studies and on information in databases. All flora were classified into 57 families and 211 species; we evaluated 253 plant communities. The representative, invasive exotic species were Sicyos angulatus, Lactuca scariola, Ambrosia trifida, Ambrosia artemisiifolia, and Eupatorium rugosum. Stands of $A$. trifida ranged in area from 214 to $16,882 \mathrm{~m}^{2}$ and were present in riparian zones, road and forest edges, slopes, and other open habitats at all sites, covering an average of $37.87 \%$ of all study areas. In Gwangju, Ansan, and Anyang cities and in Yeoncheon-gun, A. trifida coverage was $>50 \%$; the total mean coverage was near-continuous along the Han and the South Han rivers, broken only by cliffs in some riparian zones, and anthropogenic constructions. A. trifida and S. angulatus require careful management because of extensive growth, shading, and twining. The biodiversity of native species may be conserved by physical eradication of these plants. We describe the extents and distribution patterns of these two representative invasive exotic species in the enormous urban ecosystem of Seoul.
\end{abstract}

Keywords: Ambrosia trifida, exotic, invasive, management, Sicyos angulatus, spreading, urban ecosystem

\section{INTRODUCTION}

Humans have either deliberately or accidentally dispersed many exotic species outside their native ranges. Exotic species compete with native species and threaten ecosystem stability. Exotic species have greatly weakened ecosystems worldwide, as they are associated with diseases that spread among crops and forests [Krebs 2009]. We use "exotic" to define a species of foreign origin that has invaded the Korean Peninsula.

Many countries have listed their exotic species and study and manage them extensively. The Korean Ministry of Environment has created rules for the control of exotic species. However, ecological studies are very few in number, and evaluations of present ecological conditions and the impacts of exotic species on ecosystems are needed. The Korean Ministry of Environment has listed a total of 1,109 problematic species, of which exotic plants number 309 [NIE 2016]. The Ministry has identified 12 exotic species as legally disturbing ecosystems; these are noxious species. When animals are included, the problematic exotic species total 18 .

Plants in this category include $A$. trifida (from North America) and S. angulatus. A. trifida grows to $>2 \mathrm{~m}$ in height and have high population densities. This species was first recorded in northern Kyounggi during the 1970s and is now widely distributed in the riparian zones of streams and rivers and on road edges. Local officials report that $A$. trifida is expanding from forest edges into forest interiors in northern Kyounggi. A. trifida 
occurs nationwide, including in northwest Gangwon, adjacent to the Seoul metropolitan area [Choi et al. 2007]. Even in North America, A. trifida is recorded as a problem weed, outcompeting crops [Page and Nurse 2015].

$S$. angulatus (from North America; annual dendril grass) is dominant in the riparian zone, establishing areas of monoculture. This species has dispersed rapidly along the riparian zone of the Han river and its tributaries. S. angulatus grows well in soil, with about $10 \%(\mathrm{w} / \mathrm{w})$ water, under high or moderate shade conditions $(60 \%)$ [Oh et al. 2015].

Our objective was to explore the distribution of A. trifida and S. angulatus in the Seoul metropolitan area and to suggest how these very invasive exotic species might be managed.

\section{MATERIALS AND METHODS}

\section{Study area}

We analyzed databases and the relevant literature to determine the distribution, spread, and damage caused to ecosystems identified by the Ministry of Environment by exotic plants. We analyzed reports prepared by the Ministry and local governments in the Seoul metropolitan area and selected 197 potential study sites. We reduced this number to 52 by considering local administrative units, distribution areas, and site accessibility (Figure 1, Table 1). The 52 sites were located in 20 local administrative units of the Gyeonggi-do region: Goyang-si, Gwachon-si, Gwangju-si, Gunpo-si, Namyangju-si, Sungnamsi, Suwon-si, Siheung-si, Ansung-si, Ansan-si, Anyang-si, Yangpyeong-gun, Yeoju-si, Yeonchon-gun, Yongin-si, Ichon-si, Paju-si, Pyeongtaek-si, Hanam-si, Hwasung-si, and metropolitan Seoul. Most study sites were urban and rural streams. Demilitarized zones were included in the Paju-si study sites.

The $S$. angulatus study sites were riparian zones along the main Han River of Gangdonggu and Seoul, running to the South Han River of Gwangju-si and Yeoju-si. The total distance studied was approximately $88 \mathrm{~km}$. Of the $S$. angulatus study sites, 15 were beside the Han River, 2 were

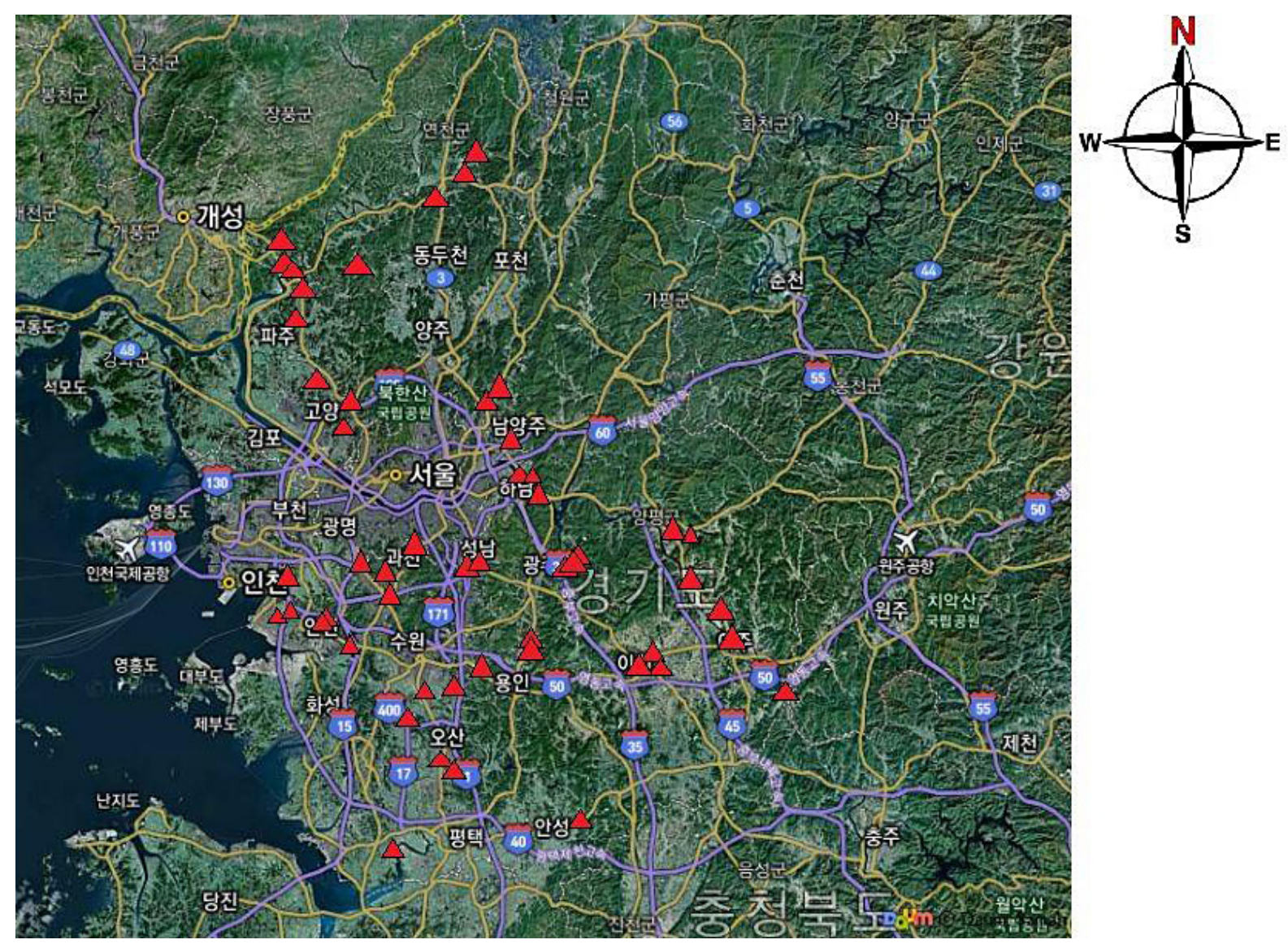

Figure 1. Study sites. The triangles indicate 52 study sites in Seoul metropolitan area where vegetation and cover survey were conducted (May 1, 2015 - Oct 31, 2016). Refer to Table 1 for details of 52 study sites 
Table 1. Distribution area of exotic plants disturbing ecosystem in study sites (-: minimal area of exotic plants disturbing ecosystem)

\begin{tabular}{|c|c|c|c|c|}
\hline \# & Address & $\begin{array}{c}\text { Exotic plants disturbing } \\
\text { ecosystem }\end{array}$ & $\begin{array}{c}\text { Distribution area } \\
\left(\mathrm{m}^{2}\right)\end{array}$ & $\begin{array}{l}\text { Total survey } \\
\text { area }\left(\mathrm{m}^{2}\right)\end{array}$ \\
\hline 1 & $\begin{array}{l}\text { Gwangchang bridge, Sunbawei station parking } \\
\text { lot Gwacheon-dong Gwacheon-si Gyeonggi-do }\end{array}$ & $\begin{array}{l}\text { Lactuca scariola, Ambrosia } \\
\text { trifida }\end{array}$ & - & 9,482 \\
\hline 2 & $\begin{array}{l}\text { Jiwol-ri Chowol-up Gwangju-si Gyeonggi-do } \\
\text { (Jiwol bridge) }\end{array}$ & $\begin{array}{l}\text { Ambrosia trifida, Ambrosia } \\
\text { artemisiifolia var. }\end{array}$ & - & 3,480 \\
\hline 3 & $\begin{array}{l}\text { Songjung bridge Songjung-dong Gwangju-si } \\
\text { Gyeonggi-do }\end{array}$ & $\begin{array}{l}\text { Ambrosia artemisiifolia var. } \\
\text { elatior }\end{array}$ & - & 8,795 \\
\hline 4 & $\begin{array}{l}\text { 24-0 Jiwol-ri Chowol-up Gwangju-si Gyeonggi- } \\
\text { do (Shinwol-2-bridge) }\end{array}$ & Ambrosia trifida & $\begin{array}{l}\text { Ambrosia trifida: } \\
2,903\end{array}$ & 32,998 \\
\hline 5 & $\begin{array}{l}\text { Near Samsin APT, Gunpo bridge Geumjung- } \\
\text { dong Gunpo-si Gyeonggi-do }\end{array}$ & $\begin{array}{l}\text { Lactuca scariola, Ambrosia } \\
\text { trifida, Ambrosia artemisiifolia } \\
\text { var. elatior }\end{array}$ & 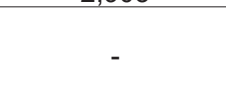 & 10,962 \\
\hline 6 & $\begin{array}{l}\text { Yatap elementary school, Yatap-10-bridge } \\
\text { Yatap-dong Bundang-gu Seongnam-si } \\
\text { Gyeonggi-do }\end{array}$ & Ambrosia trifida & - & 4,385 \\
\hline 7 & $\begin{array}{l}\text { Yeosu grand bridge Sasong-dong Sujung-gu } \\
\text { Seongnam-si Gyeonggi-do }\end{array}$ & Ambrosia trifida & - & 15,691 \\
\hline 8 & $\begin{array}{l}\text { Daehwang bridge Daehwanggyo-dong } \\
\text { Gwonsun-gu Suwon-si Gyeonggi-do }\end{array}$ & $\begin{array}{l}\text { Lactuca scariola, Ambrosia } \\
\text { trifida }\end{array}$ & - & 12,338 \\
\hline 9 & $\begin{array}{l}1959 \text { Jungwang-dong Siheung-si Gyeonggi-do } \\
\text { (Gunja-2-bridge, Gunja stream) }\end{array}$ & $\begin{array}{l}\text { Lactuca scariola, Ambrosia } \\
\text { artemisiifolia var. elatior, } \\
\text { Aster pilosus }\end{array}$ & - & 16,166 \\
\hline 10 & $\begin{array}{l}2154 \text { Jungwang-dong Siheung-si Gyeonggi-do } \\
\text { (Weigwak-4-bridge, Siheung stream) }\end{array}$ & $\begin{array}{l}\text { Lactuca scariola, Ambrosia } \\
\text { trifida, Ambrosia artemisiifolia } \\
\text { var. elatior }\end{array}$ & - & 13,800 \\
\hline 11 & Po-dong Siheung-si Gyeonggi-do (Po-ri Sluice) & $\begin{array}{l}\text { Sicyos angulatus, Lactuca } \\
\text { scariola, Ambrosia trifida, } \\
\text { Aster pilosus }\end{array}$ & $\begin{array}{l}\text { Ambrosia trifida: } \\
\quad 805\end{array}$ & 11,020 \\
\hline 12 & $\begin{array}{l}\text { Wolpi-dong Sangrok-gu Ansan-si Gyeonggi-do } \\
\text { (Ansan bridge, Ansan stream) }\end{array}$ & $\begin{array}{l}\text { Lactuca scariola, Ambrosia } \\
\text { trifida, Ambrosia artemisiifolia } \\
\text { var. elatior, Aster pilosus }\end{array}$ & - & 11,609 \\
\hline 13 & $\begin{array}{l}\text { Choji-dong Danwon-gu Ansan-si Gyeonggi-do } \\
\text { (Hwajung-1-bridge, Hwajung stream) }\end{array}$ & Ambrosia trifida & $\begin{array}{l}\text { Ambrosia trifida } \\
\quad: 2846\end{array}$ & 23,261 \\
\hline 14 & $\begin{array}{l}\text { 590-1 Palgok-2-dong Sangrok-gu Ansan-si } \\
\text { Gyeonggi-do (Palgok bridige, Banwol stream) }\end{array}$ & $\begin{array}{l}\text { Lactuca scariola, } \\
\text { Ambrosia trifida, Ambrosia } \\
\text { artemisiifolia var. elatior, } \\
\text { Aster pilosus }\end{array}$ & $\begin{array}{l}\text { Ambrosia trifida: } \\
\quad 214\end{array}$ & 13,674 \\
\hline 15 & $\begin{array}{l}\text { Naebang bridge Naebang-ri Bogae-myeon } \\
\text { Ansung-si Gyeonggi-do }\end{array}$ & Ambrosia trifida & $\begin{array}{l}\text { Ambrosia trifida } \\
: 356\end{array}$ & 11,324 \\
\hline 16 & $\begin{array}{l}\text { Bisan grand bridge Bisan-dong Dongan-gu } \\
\text { Anyang-si Gyeonggi-do }\end{array}$ & Ambrosia trifida & - & 14,688 \\
\hline 17 & $\begin{array}{l}\text { Near Seoksu station, Yeonhyun middle school } \\
\text { Seoksu-dong Manan-gu Anyang-si Gyeonggi- } \\
\text { do }\end{array}$ & $\begin{array}{l}\text { Sicyos angulatus, Lactuca } \\
\text { scariola, Ambrosia trifida, } \\
\text { Ambrosia artemisiifolia var. } \\
\text { elatior }\end{array}$ & - & 25,133 \\
\hline 18 & $\begin{array}{l}\text { Wondeog bridge Gongsae-ri Gaegun-myeon } \\
\text { Yangpyeong-gun Gyeonggi-do }\end{array}$ & $\begin{array}{l}\text { Ambrosia trifida , Ambrosia } \\
\text { artemisiifolia var. elatior }\end{array}$ & - & 34,377 \\
\hline 19 & $\begin{array}{l}\text { Infobox of Ipo-bo Ipo-ri Geumsa-ri Yangpyeong- } \\
\text { gun Gyeonggi-do }\end{array}$ & $\begin{array}{l}\text { Sicyos angulatus, Ambrosia } \\
\text { artemisiifolia var. elatior }\end{array}$ & $\begin{array}{l}\text { Sicyos angulatus } \\
: 404\end{array}$ & 30,932 \\
\hline 20 & $\begin{array}{l}\text { Hoeihyun-ri Yangpyeong-up Yangpyeong-gun } \\
\text { Gyeonggi-do }\end{array}$ & $\begin{array}{l}\text { Sicyos angulatus, } \\
\text { Ambrosia trifida, Ambrosia } \\
\text { artemisiifolia var. elatior }\end{array}$ & $\begin{array}{l}\text { Ambrosia trifida : } \\
\quad 2,496\end{array}$ & 34,641 \\
\hline 21 & $\begin{array}{l}\text { Hupo bridge Hupo-ri Daeshin-myeon Yeoju-si } \\
\text { Gyeonggi-do }\end{array}$ & $\begin{array}{l}\text { Ambrosia artemisiifolia var. } \\
\text { elatior }\end{array}$ & - & 10,876 \\
\hline 22 & Ha-ri Yeoju-up Yeoju-si Gyeonggi-do & $\begin{array}{l}\text { Ambrosia artemisiifolia var. } \\
\text { elatior }\end{array}$ & - & 18,609 \\
\hline 23 & $\begin{array}{l}\text { Near Nungnum fields Do-ri Jeomdong-myeon } \\
\text { Yeoju-si Gyeonggi-do }\end{array}$ & Sicyos angulatus & - & 26,287 \\
\hline 24 & $\begin{array}{l}\text { A bridge before confluence of Osan stream, } \\
\text { Sangha-dong Giheung-gu Yongin-si Gyeonggi- } \\
\text { do (Near Sangha elementary school) }\end{array}$ & $\begin{array}{l}\text { Lactuca scariola, Ambrosia } \\
\text { trifida }\end{array}$ & - & 2,031 \\
\hline 25 & $\begin{array}{l}\text { 447-9 Chobu-ri Mohyun-myeon Yongin-si } \\
\text { Gyeonggi-do (Shinchobu bridge) }\end{array}$ & Ambrosia trifida & $\begin{array}{l}\text { Ambrosia trifida : } \\
1,066\end{array}$ & 4,290 \\
\hline 26 & $\begin{array}{l}\text { A forked road in front of Supo bridge Cheoin-gu } \\
\text { Yongin-si Gyeonggi-do (Near Dunjeon station) }\end{array}$ & Ambrosia trifida & - & 10,653 \\
\hline 27 & $\begin{array}{l}\text { Bokha-2-bridge Galsan-dong Ichon-si } \\
\text { Gyeonggi-do }\end{array}$ & $\begin{array}{l}\text { Ambrosia trifida , Ambrosia } \\
\text { artemisiifolia var. elatior }\end{array}$ & - & 23,762 \\
\hline 28 & $\begin{array}{l}\text { Byeolmyeong bridge Jangrok-dong Ichon-si } \\
\text { Gyeonggi-do (Jangrok bridge) }\end{array}$ & $\begin{array}{l}\text { Ambrosia artemisiifolia var. } \\
\text { elatior }\end{array}$ & - & 10,962 \\
\hline 29 & $\begin{array}{l}\text { Yousan-2-bridge Jangrok-dong Ichon-si } \\
\text { Gyeonggi-do }\end{array}$ & $\begin{array}{l}\text { Ambrosia trifida, Ambrosia } \\
\text { artemisiifolia var. elatior, } \\
\text { Rumex acetocella }\end{array}$ & $\begin{array}{l}\text { Ambrosia trifida : } \\
\quad 1,793\end{array}$ & 16,965 \\
\hline
\end{tabular}




\begin{tabular}{|c|c|c|c|c|}
\hline 30 & $\begin{array}{l}\text { Geumam bridge Geumam-ri Seotan-myeon } \\
\text { Pyeongtaek-si Gyeonggi-do }\end{array}$ & $\begin{array}{l}\text { Ambrosia trifida, Aster } \\
\text { pilosus }\end{array}$ & - & 13,705 \\
\hline 31 & $\begin{array}{l}\text { Near lake Kileum-ri Osung-myeon Pyeongtaek- } \\
\text { si Gyeonggi-do }\end{array}$ & $\begin{array}{l}\text { Lactuca scariola, Sicyos } \\
\text { angulatus }\end{array}$ & - & 3,470 \\
\hline 32 & $\begin{array}{l}\text { Bongnam bridge Jinwe-myeon Pyeongtaek-si } \\
\text { Gyeonggi-do (Intersection of local road 314) }\end{array}$ & $\begin{array}{l}\text { Lactuca scariola, Ambrosia } \\
\text { trifida }\end{array}$ & - & 24,469 \\
\hline 33 & $\begin{array}{l}\text { Near Korea Water Resources Corporation } \\
\text { Baealmi-dong Hanam-si Gyeonggi-do }\end{array}$ & Eupatorium rugosum & - & 6,741 \\
\hline 34 & $\begin{array}{l}\text { Shinjang-dong Hanam-si Gyeonggi-do } \\
\text { (Deogpung stream) }\end{array}$ & Ambrosia trifida & $\begin{array}{l}\text { Ambrosia trifida: } \\
\quad 975\end{array}$ & 20,338 \\
\hline 35 & $\begin{array}{l}\text { Near Shinan APT, Sangok-2-bridge Chanwoo- } \\
\text { dong Hanam-si Gyeonggi-do (Sangok stream) }\end{array}$ & $\begin{array}{l}\text { Ambrosia trifida, Ambrosia } \\
\text { artemisiifolia var. elatior }\end{array}$ & $\begin{array}{l}\text { Ambrosia trifida : } \\
\quad 8,323\end{array}$ & 25,786 \\
\hline 36 & $\begin{array}{l}\text { Seokwoo bridge Seokwoo-ri Dongtan-myeon } \\
\text { Hwasung-si Gyeonggi-do }\end{array}$ & Ambrosia trifida & - & 20,334 \\
\hline 37 & $\begin{array}{l}\text { Yongsu bridge Goji-ri Jungnam-myeon } \\
\text { Hwasung-si Gyeonggi-do }\end{array}$ & Ambrosia trifida & - & 92,977 \\
\hline 38 & $\begin{array}{l}205-5 \text { Samsong-dong Deogyang-gu Goyang-si } \\
\text { Gyeonggi-do }\end{array}$ & Ambrosia trifida & $\begin{array}{l}\text { Ambrosia trifida: } \\
7,851\end{array}$ & 39,188 \\
\hline 39 & $\begin{array}{l}\text { 450-8 Shinwon-dong Deogyang-gu Goyang-si } \\
\text { Gyeonggi-do }\end{array}$ & $\begin{array}{l}\text { Sicyos angulatus, Lactuca } \\
\text { scariola, Ambrosia trifida, } \\
\text { Ambrosia artemisiifolia var. } \\
\text { elatior, Aster pilosus }\end{array}$ & $\begin{array}{l}\text { Ambrosia trifida: } \\
\quad 2,730\end{array}$ & 49,364 \\
\hline 40 & $\begin{array}{l}\text { 228-27 Sunghyun-ro Ilsandong-gu Goyang-si } \\
\text { Gyeonggi-do }\end{array}$ & Sicyos angulatus & $\begin{array}{c}\text { Sicyos } \\
\text { angulatus: } 1,181\end{array}$ & 11,724 \\
\hline 41 & 273-5 Ilpae-dong Namyang-si Gyeonggi-do & $\begin{array}{l}\text { Sicyos angulatus, Ambrosia } \\
\text { trifida }\end{array}$ & $\begin{array}{c}\text { Sicyos } \\
\text { angulatus: } \\
\text { 2,076; } \text { Ambrosia } \\
\text { trifida: } 771\end{array}$ & 8,470 \\
\hline 42 & $\begin{array}{l}\text { 567-24 Naegok-ri Jinjeop-up Namyang-si } \\
\text { Gyeonggi-do (Wangsuk stream) }\end{array}$ & $\begin{array}{l}\text { Sicyos angulatus, Lactuca } \\
\text { scariola, Ambrosia trifida, } \\
\text { Ambrosia artemisiifolia var. } \\
\text { elatior, Aster pilosus }\end{array}$ & $\begin{array}{c}\text { Sicyos } \\
\text { angulatus: } 648 ; \\
\text { Ambrosia trifida: } \\
2,561 \\
\end{array}$ & 5,295 \\
\hline 43 & $\begin{array}{l}\text { Shinwol bridge, Shinwol-ri Jinjeop-up } \\
\text { Namyang-si Gyeonggi-do }\end{array}$ & $\begin{array}{l}\text { Sicyos angulatus, Ambrosia } \\
\text { trifida }\end{array}$ & $\begin{array}{c}\text { Sicyos } \\
\text { angulatus: } \\
\text { 1,452; } \text { Ambrosia } \\
\text { trifida: } 2,125\end{array}$ & 5,884 \\
\hline 44 & $\begin{array}{l}518 \text { Gomun-ri Yeoncheon-up Yeoncheon-gun } \\
\text { Gyeonggi-do }\end{array}$ & $\begin{array}{l}\text { Ambrosia trifida, Aster } \\
\text { pilosus }\end{array}$ & $\begin{array}{l}\text { Ambrosia trifida: } \\
\quad 3,412\end{array}$ & 3,412 \\
\hline 45 & $\begin{array}{l}\text { San 35-10 Goneung-ri Jeongok-up Yeoncheon- } \\
\text { gun Gyeonggi-do }\end{array}$ & $\begin{array}{l}\text { Sicyos angulatus, Ambrosia } \\
\text { trifida, Aster pilosus }\end{array}$ & $\begin{array}{c}\text { Sicyos } \\
\text { angulatus: } \\
\text { 2,191; } \text { Ambrosia } \\
\text { trifida: } 7,586 \\
\end{array}$ & 14,869 \\
\hline 46 & $\begin{array}{l}\text { 13-1 Shindap-ri Jeongok-up Yeoncheon-gun } \\
\text { Gyeonggi-do }\end{array}$ & $\begin{array}{l}\text { Ambrosia trifida , Aster } \\
\text { pilosus }\end{array}$ & $\begin{array}{c}\text { Ambrosia trifida: } \\
\text { 15,725; } \\
\text { Aster pilosus: } \\
2,963 \\
\end{array}$ & 18,688 \\
\hline 47 & $\begin{array}{l}\text { 336-9 Bongseo-ri Munsan-up Paju-si } \\
\text { Gyeonggi-do }\end{array}$ & $\begin{array}{l}\text { Ambrosia trifida, Ambrosia } \\
\text { artemisiifolia var. elatior, } \\
\text { Aster pilosus }\end{array}$ & $\begin{array}{l}\text { Ambrosia trifida: } \\
\quad 5,105\end{array}$ & 18,954 \\
\hline 48 & $\begin{array}{l}687 \text { Jikcheon-ri Bupwon-up Paju-si Gyeonggi- } \\
\text { do }\end{array}$ & Ambrosia trifida & $\begin{array}{c}\text { Ambrosia trifida: } \\
11,991\end{array}$ & 11,991 \\
\hline 49 & 741-1 Bongam-ri Paju-up Paju-si Gyeonggi-do & $\begin{array}{l}\text { Sicyos angulatus, Ambrosia } \\
\text { trifida, Aster pilosus }\end{array}$ & $\begin{array}{l}\text { Ambrosia trifida: } \\
11,019\end{array}$ & 75,539 \\
\hline 50 & DMZ1, Deokjinsansung & $\begin{array}{l}\text { Sicyos angulatus, Ambrosia } \\
\text { trifida, Aster pilosus }\end{array}$ & $\begin{array}{l}\text { Ambrosia trifida: } \\
16,882\end{array}$ & 16,882 \\
\hline 51 & DMZ2 & Ambrosia trifida & $\begin{array}{c}\text { Ambrosia trifida: } \\
14,763\end{array}$ & 15,202 \\
\hline \multirow[t]{2}{*}{52} & DMZ3, Sunae stream & $\begin{array}{l}\text { Ambrosia trifida, Ambrosia } \\
\text { artemisiifolia var. elatior, } \\
\text { Aster pilosus }\end{array}$ & $\begin{array}{c}\text { Ambrosia trifida: } \\
\quad 4,511\end{array}$ & 4,511 \\
\hline & Total & & 139,724 & 970,984 \\
\hline
\end{tabular}

in Lake Paldang, and 26 were along the South Han river (Table 3).

\section{Vegetation and cover analyses}

The GPS locations, physical addresses, study areas, dominant species, and areas covered by the species were recorded in detail. We selected reference points and performed vegetation surveys in circles of radii $500 \mathrm{~m}$ from such points. We established plant community quadrats at all sites. If the area occupied by a problematic species were $>100 \mathrm{~m}^{2}$, we considered that the species formed a plant community. All species in each quadrat were recorded, and coverages were visually estimated (in terms of the dominant 
class) using the Braun-Blanquet scale [Fuller and Conard 1932].

\section{GIS analyses}

We downloaded recent geomorphological maps at a 1:25,000 scale and recorded each study site, the area thereof, and geomorphological data. We noted the proportions of annuals, perennials, shrubs and trees, exotic plants, waterways, open lots, artificial structures, forest vegetation, housing and business development, arable land, and the following 12 problematic plants: A. trifida, $A$. artemisiifolia var. elatior, E. rugosum, A. pilosus, $H$. radicata, S. altissima, L. scariolia, $P$. distichum var. distichum, P. distichum var. indutum, $S$. carolinense, S. angulatus, and R. acetosella. All areas $<100 \mathrm{~m}^{2}$ were amalgamated with adjacent areas. We used Q-GIS ver. 2.2.0 to define the areas [QGIS 2016]. All plant coverages were calculated using Q-GIS data.

\section{Statistical analyses}

All problematic plant coverages recorded were pooled to estimate and rank total mean $A$. trifida coverages in local administrative units. We employed the Kruskal-Wallis rank test of SPSS software ver. 12.0.

\section{Spread rates}

We calculated $A$. trifida and $S$. angulatus spread rates [Kil et al. 2012; Kil et al. 2013] using the following equation: Spread rate $\left(\mathrm{m}^{2} /\right.$ year $)$ $=($ area of plant species in a certain year-area in year 1)/(Arabic number of test year-Arabic number in year 1). The spread rates in similar ecosystems (open lots, riparian zones, and road edges) were averaged.

\section{RESULTS AND DISCUSSION}

\section{Vegetation analysis}

On the 197 study sites, the frequencies (in decreasing order) of exotic plants disturbing the ecosystem were: A. trifida (65\%), A. artemisiifolia var. elatior (54\%), A. pilosus (30\%), S. angulatus (23\%), L. scariolia (8\%), and E. rugsom $(3 \%)(n=197)$. The quadrat survey showed that 211 species of 57 families were involved. We recorded 50 problematic plant communities among the 253 communities (Table 2). The dominant problematic species were $S$. angulatus, $L$. scariola, A. trifida, A. artemisiifolia var. elatior, and $E$. rugosum. The most frequent species encountered in the vegetation survey was $A$. trifida, whereas the most common native species were Pragmites communis and Pragmites japonica. The dominant exotic species of plant communities that did not disturb the ecosystem were Ailanthus altissima, Erigeron annuus, Oenothera biennis, Helianthus tuberosus, Erigeron canadensis, Bromus richardsonii, Robinia pseudoacacia, Amorpha fruticose, Chenopodium serotinum, Lepidium virginicum, Coreopsis lanceolate, Festuca ovina, Trifolium repens, and Chenopodium album. Among native species, perennials and annuals dominated; most exotic and problematic exotic species were adaptable annuals (Figure 2), particularly in the riparian zones of the Han and South Han Rivers (Figure 3).

\section{Distributional status of $A$. trifida and S. angulatus}

The ratio of the area of exotic plants disturbing the ecosystem to that of all study sites was ca. $14 \%$ (Table 1). The A. trifida distributional areas ranged from 214 (590-1 Palgok-2-dong Sangrok-gu Ansan-si Gyeonggi-do; Palgok Bridge, Banwol Stream) to $16,882 \mathrm{~m}^{2}$ (DMZ, Deokjinsansung) (Table 1). We recorded 37 S. angulatus communities on the principal streams of the Han and South Han Rivers (Tables 3, 4). Quadrat vegetation analysis revealed 44 species in 24 families among 3 plant communities. The areas of all but the principal $S$. angulatus communities ranged from $20.53 \mathrm{~m}^{2}$ to $244,248.89 \mathrm{~m}^{2}$ (Table 3). The largest area of $S$ angulatus was 434,903 $\mathrm{m}^{2}$, constituting an epicenter of $S$. angulatus propagation. This community was located at the largest cape of the Han River; upstream Sicyos angulatus seeds are delivered when the water velocity is low; they then germinate and grow. S. angulatus propagation is influenced by the direction of upstream flow [Osawa et al. 2013]. The plant communities recorded along the Han and South Han Rivers were $S$. angulatus, $A$. princeps var. orientalis, and Humulus japonicas (Table 4); S. angulatus was the most common (95\%).

\section{Spread rates of A. trifida and S. angulatus}

The $A$. trifida spread rate ranged from 4,972 $\mathrm{m}^{2} /$ year to $337,500 \mathrm{~m}^{2} /$ year (Table 6 ), and it was 
Table 2. Status of plant communities appeared in study sites. The number in parenthesis indicates frequency recorded. No number represents one frequency

\begin{tabular}{|c|c|c|}
\hline Name of plant community & $\begin{array}{c}\text { Number of plant } \\
\text { community }\end{array}$ & Locations(Refer to number of locations in Table 1) \\
\hline Sicyos angulatus ${ }^{\mathrm{a}}$ & 14 & $11,19,20(2), 31,39(3), 40(3), 43,45,49$ \\
\hline Lactuca scariola $^{a}$ & 5 & $10,12,14(2), 31$ \\
\hline Ailanthus altissima & 1 & 8 \\
\hline Phragmites communis & 16 & $3,8,10,11,15,21,26,27,28,29,30,31,32(2), 36,37$ \\
\hline Phalaris arundinacea & 3 & $5,14,25$ \\
\hline Setaria viridis & 2 & 41,42 \\
\hline Erigeron annuus & 7 & $2,4,5,7,8,29,35$ \\
\hline Agropyron tsukushiense var. transiens & 2 & 1,37 \\
\hline Salix gracilistyla & 2 & 6,23 \\
\hline Persicaria thunbergii & 12 & $1(2), 2,3,4,5,12,13,21,24,43,49$ \\
\hline Salix pseudolasiogyne & 4 & $5,16,26,37$ \\
\hline Ambrosia trifida ${ }^{a}$ & 39 & $\begin{array}{l}1,2,4(2), 5,7,8(2), 12(2), 13(3), 14(4), 16,17(2), 18,20(2), \\
24(2), 25(3), 26,27,29,34,35(2), 36,37(3), 38(5), 39(2), \\
41(1), 42(2), 44(2), 45(1), 46(2), 47(3), 48,49(6)\end{array}$ \\
\hline Oenothera biennis & 3 & $23,26(2)$ \\
\hline Phragmites japonica & 22 & $\begin{array}{l}1,2,4,6,7,9,11,12,13,14,16,17,18,19,20,23,34,35 \text {, } \\
39,42,43,45\end{array}$ \\
\hline Glycine soja & 1 & 38 \\
\hline Echinochloa crusgalli & 1 & 41 \\
\hline Ambrosia artemisiifolia var. elatior ${ }^{a}$ & 3 & $10,14,19$ \\
\hline Helianthus tuberosus & 1 & 30 \\
\hline Erigeron canadensis & 2 & 12,35 \\
\hline Scirpus fluviatilis & 1 & 22 \\
\hline Artemisia selengensis & 2 & 22,26 \\
\hline Miscanthus sacchariflorus & 12 & $1,5,7,11,12,16,17,18(2), 34,47,49$ \\
\hline Aster pilosus & 1 & 47 \\
\hline Digitaria sanguinalis & 1 & 36 \\
\hline Salix koreensis & 5 & $1,20,30,32,36$ \\
\hline Lespedeza cuneata & 2 & 19,38 \\
\hline Bromus richardsonii & 5 & $9,15,16,30,37$ \\
\hline Rubus crataegifolius & 1 & 25 \\
\hline Umbelliferae spp. & 1 & 31 \\
\hline Eupatorium rugosum ${ }^{\mathrm{a}}$ & 2 & $33(2)$ \\
\hline Salix subfragilis & 5 & $1,16,17,18,34$ \\
\hline Agropyron ciliare & 1 & 17 \\
\hline Equisetum arvense & 5 & $6,21,27,28,30$ \\
\hline Artemisia princeps var. orientalis & 8 & $4,6,10,14,32,39,45(2)$ \\
\hline Robinia pseudoacacia & 13 & $4,14,15,17,20,27,28,29,32,37,38,39,42$ \\
\hline Chelidonium majus var. asiaticum & 5 & $2,15,20,27,29$ \\
\hline Salix chaenomeloides & 1 & 7 \\
\hline Amorpha fruticosa & 1 & 36 \\
\hline Chenopodium serotinum & 1 & 7 \\
\hline Zizantia latifolia & 3 & $6,7,14$ \\
\hline Pueraria thunbergiana & 11 & $1,3,14,18,19,26,35,40,42,45,47$ \\
\hline Lepidium virginicum & 2 & $16(2)$ \\
\hline Persicaria nodosa & 2 & 3,14 \\
\hline Coreopsis lanceolata & 2 & 19,34 \\
\hline Festuca ovina & 7 & $6,9,13,16,35,37(2)$ \\
\hline Trifolium repens & 1 & 30 \\
\hline Poa spp. & 1 & 29 \\
\hline Platanus occidentalis & 1 & 16 \\
\hline Humulus japonicus & 7 & $1,5,9,19,27,28,40$ \\
\hline Chenopodium album & 3 & $22,24,25$ \\
\hline Total & 253 & \\
\hline
\end{tabular}

a: Exotic plants disturbing ecosystem. 


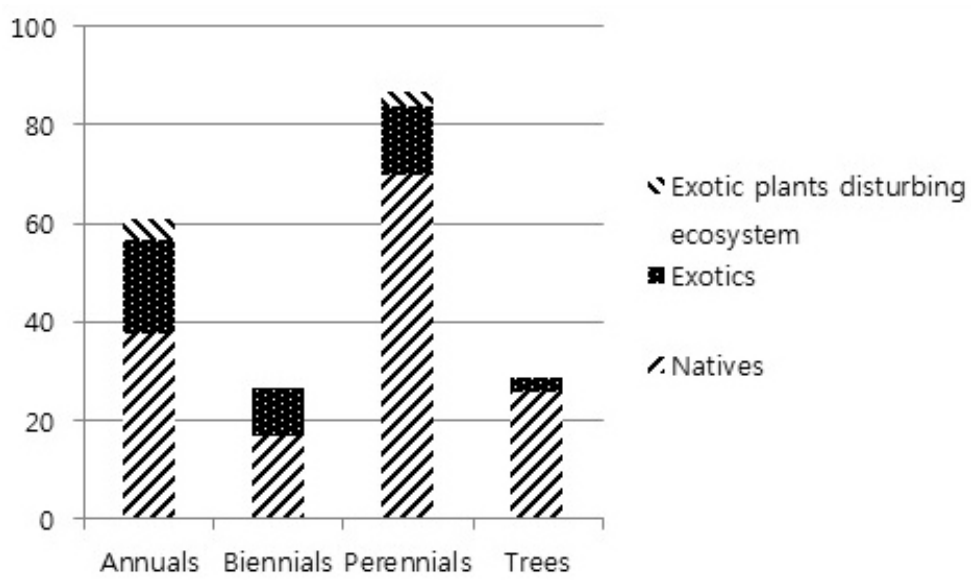

Figure 2. Life forms of flora in study sites

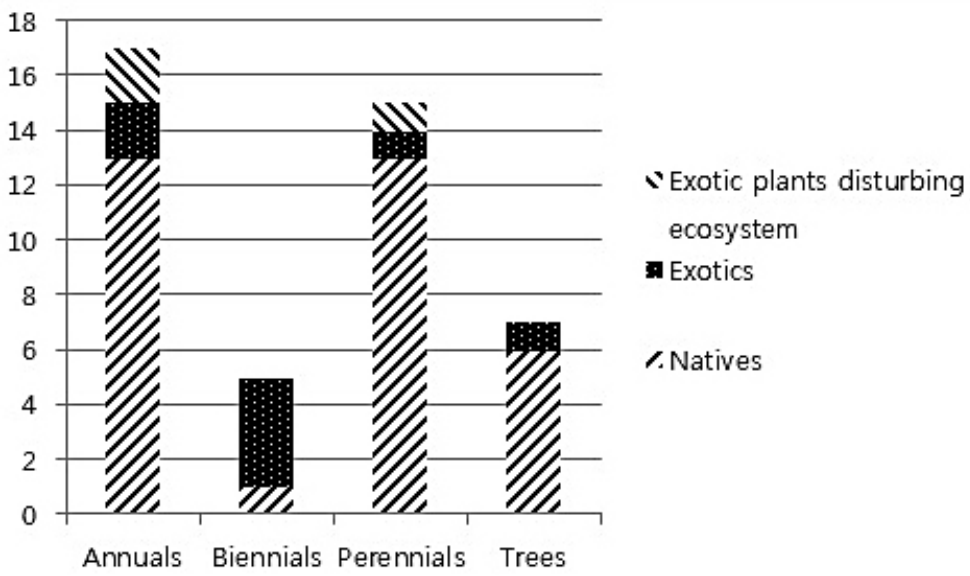

Figure 3. Life forms of flora in the Han and the South Han river

lowest in the riparian zone. However, A. trifida is spread by flowing water [Kim and Choi 2008] and may invade adjacent sites. The spread rate was higher along road edges than in riparian zones, although the species disturbs and damages Korean river ecosystems [Seo et al. 2012]. S. angulatus was found principally along riversides and on small mountains, roadsides, and open and cultivated areas [Moon et al. 2008; Kim et al. 2014]. The coverages of these two species fell over time at some sites, possibly due to construction and changes in land use.

\section{Management of A. trifida and S. angulatus}

We used the species coverage data to analyze the distribution densities of exotic plants disturbing the ecosystem. However, not all the 12 such species were represented in the vegetation survey. Only A. trifida was found at all study sites (37.87\% mean coverage; Table 5). Coverage was $>50 \%$ in Gwangju-si, Ansan-si, Anyang-si, and
Yeoncheon-gun. The Kruskal-Wallis test showed that the A. trifida coverage differed significantly from that of other plants $(p<0.05$; Table 5).

A. trifida was the most common exotic species and was found in small or large patches in the Seoul metropolitan area. This species can invade forest edges and interiors, the riparian zones of urban streams, and disturbed sites. The shading effect of $A$. trifida negatively affects native species; in the Seoul metropolitan area, A. trifida can grow to about $3 \mathrm{~m}$ in height prior to September/October. A. trifida dominated the DMZ of Paju City, forming monocultures in fields by road edges, abandoned paddy fields, and military training areas. This may be explained by the fact that the seeds are spread by the transportation of soldiers and equipment. We recommend that military transports and uniforms be cleaned after training exercises.

The distance between $S$. angulatus patches downstream near Hanam-si was shorter that that upstream near Yeoju-si (Figure 4), which is ex- 
Table 3. Study sites for Sicyos angulatus in the Han river and The South Han river of the distance about 88.75 km along Gangdong-gu to Samhap-ri Jumdong-myeon Yeoju-si (8-1 to 8-7 sites represents one connected patches dominated by Sicyos angulatus)

\begin{tabular}{|c|c|c|c|c|c|}
\hline No. & Address & Longitude & Latitude & $\begin{array}{l}\text { Name of } \\
\text { river }\end{array}$ & $\begin{array}{c}\text { Area of } \\
\text { Sicyos } \\
\text { angulatus } \\
\left(\mathrm{m}^{2}\right)\end{array}$ \\
\hline 1 & 351-1 Chonho-2-dong Gandong-gu Seoul & $1270712 \mathrm{E}$ & $373250 \mathrm{~N}$ & Han & 230.53 \\
\hline 2 & 508-2 Chonho-2-dong Gandong-gu Seoul & $1270708 \mathrm{E}$ & $373253 \mathrm{~N}$ & Han & $67,294.18$ \\
\hline 3 & 661-1 Amsa-2-dong Gangdong-gu Seoul & $1270723 \mathrm{E}$ & $373323 \mathrm{~N}$ & Han & 998.23 \\
\hline 4 & 660 Amsa-2-dong Gangdong-gu Seoul & $1270725 \mathrm{E}$ & $373327 \mathrm{~N}$ & Han & $12,471.49$ \\
\hline 5 & 166-8 Amsa-2-dong Gangdong-gu Seoul & $1270739 \mathrm{E}$ & $373341 \mathrm{~N}$ & Han & $4,444.93$ \\
\hline 6 & 148-13 Amsa-2-dong Gangdong-gu Seoul & $1270746 \mathrm{E}$ & $373344 \mathrm{~N}$ & Han & $10,377.04$ \\
\hline 7 & 378 Godeug-dong Gangdong-gu Seoul & $1270918 \mathrm{E}$ & $373413 \mathrm{~N}$ & Han & 1251.91 \\
\hline $8-1$ & 14 Gangil-dong Gangdong-gu Seoul & $1271007 \mathrm{E}$ & $373442 \mathrm{~N}$ & Han & $10,229.28$ \\
\hline $8-2$ & $\begin{array}{l}399 \text { Sun-dong Hanam-si Kyeonggi-do } \\
\text { Gyeonggi-do }\end{array}$ & 12711 09E & $373457 \mathrm{~N}$ & Han & $244,248.89$ \\
\hline $8-3$ & 78 Misa-dong Hanam-si Gyeonggi-do & $1271249 E$ & $373353 \mathrm{~N}$ & Han & $4,413.47$ \\
\hline $8-4$ & Dangjung-dong Hanam-si Gyeonggi-do & $1271306 \mathrm{E}$ & $373330 N$ & Han & $74,801.58$ \\
\hline $8-5$ & 2-9 Misa-dong Hanam-si Gyeonggi-do & $1271315 \mathrm{E}$ & $373309 \mathrm{~N}$ & Han & $1,362.99$ \\
\hline $8-6$ & 516 Changwoo-dong Hanam-si Gyeonggi-do & $1271336 \mathrm{E}$ & $373255 \mathrm{~N}$ & Han & $99,846.91$ \\
\hline $8-7$ & San 2-22 Baealmi-dong Hanam-si Gyeonggi-do & $1271440 \mathrm{E}$ & $373223 \mathrm{~N}$ & Han & 1897.44 \\
\hline 9 & 159-18 Baealmi-dong Hanam-si Gyeonggi-do & 12715 07E & $373215 \mathrm{~N}$ & Han & 2436.71 \\
\hline 10 & 657-2 Bunwon-ri Namjong-myeon Gwangju-si & $1271814 \mathrm{E}$ & $373003 \mathrm{~N}$ & $\begin{array}{l}\text { Lake } \\
\text { Paldang }\end{array}$ & 1347.77 \\
\hline 11 & 198-3 Sucheong-ri Namjong-myeon Gwangju-si & 12722 43E & $373010 \mathrm{~N}$ & South Han & 1475.78 \\
\hline 12 & $\begin{array}{l}\text { 285-2 Unsim-ri Gangha-myeon Yangpyeong- } \\
\text { gun Gyeonggi-do }\end{array}$ & $1272327 \mathrm{E}$ & $372935 \mathrm{~N}$ & South Han & 2690.89 \\
\hline 13 & $\begin{array}{l}\text { 68-5 Unsim-ri Gangha-myeon Yangpyeong-gun } \\
\text { Gyeonggi-do }\end{array}$ & $1272430 \mathrm{E}$ & $372949 N$ & South Han & 749.90 \\
\hline 14 & $\begin{array}{l}\text { 1049-2 Jeonsu-ri Gangha-myeon Yangpyeong- } \\
\text { gun Gyeonggi-do }\end{array}$ & $1272455 \mathrm{E}$ & $373002 \mathrm{~N}$ & South Han & 1243.36 \\
\hline 15 & $\begin{array}{l}\text { 1038-1 Jeonsu-ri Gangha-myeon Yangpyeong- } \\
\text { gun Gyeonggi-do }\end{array}$ & $1272452 \mathrm{E}$ & $372954 \mathrm{~N}$ & South Han & 600.03 \\
\hline 16 & $\begin{array}{l}\text { 41-6 Geumsa-ri Geumsa-myeon Yeoju-si } \\
\text { Gyeonggi-do }\end{array}$ & $1273142 \mathrm{E}$ & $372516 \mathrm{~N}$ & South Han & 9917.70 \\
\hline 17 & $\begin{array}{l}241 \text { Geumsa-ri Namjong-myeon Gwangju-si } \\
\text { Gyeonggi-do }\end{array}$ & $1271820 \mathrm{E}$ & $372918 \mathrm{~N}$ & $\begin{array}{l}\text { Lake } \\
\text { Paldang }\end{array}$ & 319.56 \\
\hline 18 & $\begin{array}{l}350-2 \text { Gyopyeong-ri Gangsang-myeon } \\
\text { Yangpyeong-gun Gyeonggi-do }\end{array}$ & $1272916 \mathrm{E}$ & $372905 \mathrm{~N}$ & South Han & 2417.94 \\
\hline 19 & $\begin{array}{l}232 \text { Byeongsan-ri Gangsang-myeon } \\
\text { Yangpyeong-gun Gyeonggi-do }\end{array}$ & $1272806 \mathrm{E}$ & $372944 \mathrm{~N}$ & South Han & 175.51 \\
\hline 20 & $\begin{array}{l}\text { San166-5 Byeongsan-ri Gangsang-myeon } \\
\text { Yangpyeong-gun Gyeonggi-do }\end{array}$ & $1272711 \mathrm{E}$ & $373003 \mathrm{~N}$ & South Han & 1990.28 \\
\hline 21 & $\begin{array}{l}479 \text { Jeonsu-ri Gangha-myeon Yangpyeong-gun } \\
\text { Gyeonggi-do }\end{array}$ & 12725 54E & $373019 \mathrm{~N}$ & South Han & 1531.23 \\
\hline 22 & $\begin{array}{l}\text { San } 23 \text { Sangbaek-ri Heungchon-myeon Yeoju-si } \\
\text { Gyeonggi-do }\end{array}$ & $1273341 \mathrm{E}$ & $372136 \mathrm{~N}$ & South Han & 25.48 \\
\hline 23 & $\begin{array}{l}\text { 177-3 Sangbaek-ri Heungchon-myeon Yeoju-si } \\
\text { Gyeonggi-do }\end{array}$ & $1273320 \mathrm{E}$ & $372143 \mathrm{~N}$ & South Han & 839.73 \\
\hline 24 & $\begin{array}{l}164 \text { Sangbaek-ri Heungchon-myeon Yeoju-si } \\
\text { Gyeonggi-do }\end{array}$ & $1273310 \mathrm{E}$ & $372154 \mathrm{~N}$ & South Han & 20.53 \\
\hline 25 & $\begin{array}{l}36-10 \text { Gyeshin-ri Heungchon-myeon Yeoju-si } \\
\text { Gyeonggi-do }\end{array}$ & 12732 17E & $372310 \mathrm{~N}$ & South Han & 732.02 \\
\hline 26 & $\begin{array}{l}\text { 318-2 Dangnam-ri Daeshin-myeon Yeoju-si } \\
\text { Gyeonggi-do }\end{array}$ & 12732 09E & $372326 \mathrm{~N}$ & South Han & 1559.16 \\
\hline 27 & 2 Samhap-ri Jumdong Yeoju-si Gyeonggi-do & $1274449 E$ & $371326 \mathrm{~N}$ & South Han & 130.53 \\
\hline 28 & 132 Do-ri Jumdong Yeoju-si Gyeonggi-do & $1274233 \mathrm{E}$ & $371337 \mathrm{~N}$ & South Han & 5586.18 \\
\hline 29 & 202-1 Uman-ri Yeoju-up Yeoju-si Gyeonggi-do & $1274050 \mathrm{E}$ & $371505 \mathrm{~N}$ & South Han & 219.76 \\
\hline 30 & 49-3 Danhyun-ri Yeoju-up Yeoju-si Gyeonggi-do & $1274058 \mathrm{E}$ & $371626 \mathrm{~N}$ & South Han & 219.76 \\
\hline 31 & 305 Yeonyang-ri Yeoju-up Yeoju-si Gyeonggi-do & $1274007 \mathrm{E}$ & $371743 \mathrm{~N}$ & South Han & 129.67 \\
\hline 32 & 39 Janae-ro Yeoju-up Yeoju-si Gyeonggi-do & $127392.96 \mathrm{E}$ & $371736 \mathrm{~N}$ & South Han & 844.05 \\
\hline 33 & 136-10 Sang-ri Yeoju-up Yeoju-si Gyeonggi-do & $1273852 \mathrm{E}$ & $371743 \mathrm{~N}$ & South Han & 3055.97 \\
\hline 34 & 3-108 Ha-ri Yeoju-up Yeoju-si Gyeonggi-do & $1273710 \mathrm{E}$ & $371819 \mathrm{~N}$ & South Han & 199.00 \\
\hline
\end{tabular}




\begin{tabular}{|c|l|l|l|l|l|}
\hline 35 & $\begin{array}{l}\text { 1-1 Wangdae-ri Neungseo-myeon Yeoju-si } \\
\text { Gyeonggi-do }\end{array}$ & $1273703 \mathrm{E}$ & $371850 \mathrm{~N}$ & South Han & 314.26 \\
\hline 36 & $\begin{array}{l}397-10 \text { Wangdae-ri Neungseo-myeon Yeoju-si } \\
\text { Gyeonggi-do }\end{array}$ & $1273558 \mathrm{E}$ & $371946 \mathrm{~N}$ & South Han & 864.38 \\
\hline 37 & $\begin{array}{l}\text { 59-1 Naeyang-ri Neungseo-myeon Yeoju-si } \\
\text { Gyeonggi-do }\end{array}$ & $1273423 \mathrm{E}$ & $372116 \mathrm{~N}$ & South Han & 814.97 \\
\hline
\end{tabular}

Table 4. Status of plant communities at riparian zones of the Han and the South Han river

\begin{tabular}{|c|c|c|}
\hline Name of plant communities & $\begin{array}{l}\text { Number of plant } \\
\text { communities }\end{array}$ & Sites(refer to name of sites in Table3) \\
\hline Sicyos angulatus ${ }^{a}$ & (om & $\begin{array}{l}1,2,3,4,5,6,7,8-1,8-2,8-3,8-4,8-5,8-6,8-7,9,10,11, \\
12,13,15,16,17,18,19,20,21,23,24,25,26,27,28,29,30 \\
31,32,33,34,35,36,37\end{array}$ \\
\hline Artemisia princeps var. orientalis & 1 & 然 \\
\hline Humulus japonicus & 1 & 14 \\
\hline Total & 37 & \\
\hline
\end{tabular}

a Exotic plants disturbing ecosystem

Table 5. Total mean cover of exotic plant disturbing ecosystem in local cities of Seoul metropolitan area $( \pm \mathrm{SD} ;-$ means no data). The different superscript numbers represent significant rank of means by Kruskal-Wallis Test

\begin{tabular}{|c|c|c|c|c|c|c|}
\hline Cities & $\begin{array}{c}\text { Sicyos } \\
\text { angulatus }\end{array}$ & $\begin{array}{l}\text { Lactuca } \\
\text { scariola }\end{array}$ & Ambrosia trifida & $\begin{array}{c}\text { Ambrosia } \\
\text { artemisiifolia var. } \\
\text { elatior }\end{array}$ & Aster pilosus & Rumex acetocella \\
\hline Goyang-si & $20.71 \pm 31.28$ & - & $24.77^{17)} \pm 32.26$ & $0.00 \pm 0.02$ & $2.00 \pm 6.93$ & - \\
\hline Gwacheon-si & - & - & $26.18^{13)} \pm 19.99$ & - & - & - \\
\hline Gwangju-si & - & - & $67.51^{11} \pm 32.27$ & 17.5 & - & - \\
\hline Gunpo-si & - & 0.05 & $30.76^{12)} \pm 40.84$ & - & - & - \\
\hline Namyangju-si & $13.08 \pm 29.83$ & $0.15 \pm 0.55$ & $18.85^{19)} \pm 34.89$ & - & $0.38 \pm 1.39$ & - \\
\hline Seongnam-si & - & - & $26.67^{11)} \pm 7.64$ & - & - & - \\
\hline Suwon-si & - & - & $70^{2)}$ & - & - & - \\
\hline Siheung-si & $38.75 \pm 22.5$ & 55 & $32.89^{16)} \pm 34.95$ & $15 \pm 9.35$ & 18 & - \\
\hline Ansan-si & - & $18.91 \pm 17.61$ & $60^{3)} \pm 31.21$ & 46.7 & 7.68 & - \\
\hline Anseong-si & - & - & $45^{10)}$ & - & - & - \\
\hline Anyang-si & 20 & - & $55.83^{5)} \pm 26.72$ & - & - & - \\
\hline Yangpyeong-gun & $19.72 \pm 28.83$ & - & $34.04^{15)} \pm 41.74$ & 35.03 & - & - \\
\hline Yeoju-si & - & - & $0.05^{20)}$ & 0.05 & - & - \\
\hline Yeoncheon-gun & $12.73 \pm 28.67$ & - & $55.45^{4)} \pm 32.97$ & - & $4.12 \pm 10.67$ & - \\
\hline Yongin-si & - & - & $49.42^{7} \pm 37.62$ & - & - & - \\
\hline Icheon-si & 0.1 & 10 & $49.5^{6} \pm 42.79$ & 4.68 & - & 40 \\
\hline Paju-si & $4.33 \pm 16.78$ & - & $48.80^{8)} \pm 28.60$ & $0.01 \pm 0.03$ & $10.60 \pm 18.53$ & - \\
\hline Pyeongtaek-si & 70 & 60 & $10.03^{18)} \pm 14.11$ & - & - & - \\
\hline Hanam-si & - & - & $32.02^{14)} \pm 35.38$ & 40 & - & - \\
\hline Hwasung-si & - & - & $45^{9} \pm 34.93$ & - & - & - \\
\hline Total mean cover & $8.75 \pm 22.22$ & $1.98 \pm 8.91$ & $37.87 \pm 34.67$ & $2.50 \pm 11.14$ & $1.95 \pm 7.74$ & 40 \\
\hline
\end{tabular}

plained by the fact that construction affected the former riparian zone. Under such circumstances, $S$. angulatus may spread from the principal streams along tributaries, including the waterways of paddy fields and ditches. Thus, tributary populations must be managed. In terms of biodiversity conservation, $A$. trifida and $S$. angulatus must be eliminated; the former plant grows rapidly and densely, and the latter is associated with shading and dendrilling. Exotic plants disturbing ecosystems can be harvested and turned into biological resources, such as nitrogen fertilizers [Kim et al. 2016]. Also, they may serve as educational materials in terms of plant classification and observation. Exotic plants should be sustainably used rather than dumped as organic waste.

\section{Acknowledgments}

We would like to provide sincere thanks to the supports given by Gyeonggi, Seoul and Ansan Green Environmental Center. 
Table 6. Status of spreading of Ambrosia trifida and Sicyos angulatus (Value \pm SD)

\begin{tabular}{|c|c|c|}
\hline EPDE & Ecosystem type & Spreading rate $\left(\mathrm{m}^{2} / \mathrm{y}\right)$ \\
\hline \multirow{3}{*}{ Ambrosia trifida } & Open lots & 337,500 \\
& Road edge & 23,333 \\
\cline { 2 - 3 } & Riparian zone & $4,972 \pm 3,354$ \\
\cline { 2 - 3 } & Road edge & $3,125 \pm 2,839$ \\
\hline \multirow{2}{*}{ Sicyos angulatus } & Riparian zone & 262 \\
\cline { 2 - 3 } & & \\
\cline { 2 - 3 }
\end{tabular}

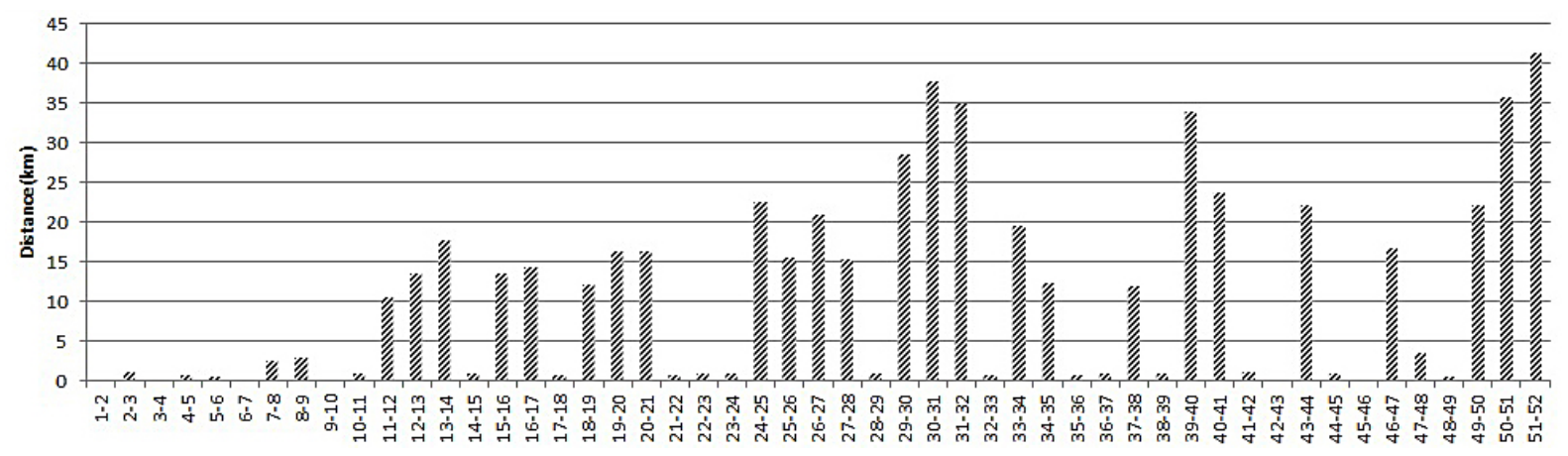

Figure 4. The distances between patches of Sicyos angulatus at the riparian zones of the Han and the South Han river. The number indicates study sites (1-2: Gangdong-gu, Seoul 51-52: Jumdong-myeon, Yeoju-si)

\section{REFERENCES}

1. Choi H.J., Lim S.H., Kim K.H., Kim S. 2007. Distribution of giant ragweed (Ambrosia trifida L.) at northwest of Gangwon, Korea. Kor J Weed Sci, 27 , 241-247.

2. Fuller G.D., Conard H.S. 1932. Plant sociology (Authorized English translation of Pflanzensoziologie by Dr. Braun-Blanquet J). McGraw-Hill Book Company, New York.

3. Kil J.H., Kim Y.H., Kim D.E., Kim H.M., Lee D.H., Hwang S.M., Kim S.Y., Lee J.C., Baek W.G., Park H.J., Oh H.K., Park K.U., Cho Y.B., Oh G.S. 2013. Monitoring of invasive alien species designated by the wildlife protection act (VII). National Institute of Environmental Research, Incheon.

4. Kil J.H., Kim Y.H., Kim D.E., Kim H.M., Lee D.H., Hwang S.M., Lee C.W., Yang B.G. 2012. Monitoring of invasive alien species designated by the wildlife protection act (VI). National Institute of Environmental Research, Incheon.

5. Kim C-S, Lee I-Y, Lee J-r, Hong S-H, Oh Y-J. 2014. Distribution of exotic weeds on upland crop field in Gyeonggi-do. Weed Turf Sci, 3, 284-291.

6. Kim M., Min H., Hong S., Kim J-G. 2016. The applicability of burcucumber (Sicyos angulatus L.) as a substitute for nitrogen fertilizer. Korean J Environ Agric, 35, 1-5.

7. Kim S., Choi H-J. 2008. Distribution of giant ragweed (Ambrosia trifida L.) at Yangu, Gangwon-do, Korea. Kor J Weed Sci, 28, 242-247.
8. Krebs C.J. 2009. Ecology, 6th ed. Benjamin Cummings, Cape Town.

9. Moon B. C., Oh S. M., Lee I. Y., Kim C. S., Cho J. R., Kim S.C. 2008. Change of weed species in Burcucumber (Sicyos angulatus L.) community and domestic distribution aspect. Kor J Weed Sci, 28, 117-125.

10. NIE(National Institute of Ecology). 2016. Information of Korean Alien Species. National Institute of Ecology. http://kias.nie.re.kr/alienspecies/ Accessed 16 June 2016.

11. Oh D., Shim D., Song S., Oh J., Hong S., Shim S. 2015. Effects of soil moisture condition and shading on growth of invasive plant Burcucumber (Sicyos angulatus L.). Weed Turf Sci, 4, 315-320.

12. Osawa T., Mitsuhashi H., Niwa H. 2013. Many alien invasive plants disperse against the direction of stream flow in riparian areas. Ecol Complex, 15, 26-32.

13. Page E.R., Nurse R.E. 2015. Cropping systems and the prevalence of giant ragweed (Ambrosia trifida): From the 1950's to present. Field Crop Res, 184, 104-111.

14. QGIS. 2016. Documentation QGIS 2.2. QGIS http:// http://docs.qgis.org/2.2/ko/docs/training_ manual/foreword/index.html. Accessed 15 May 2016.

15. Seo J., Park K-H, You J-H. 2012. Vascular plants around Seomjin river estuary in Hadong-gun, Gyeongsangnam-do. J Korean Inst For Recreation, 16, 35-49. 